\title{
Optimal multihump filter for photometric redshifts
}

\author{
Tamás Budavári ${ }^{1}$, Alexander S. Szalay \\ Department of Physics and Astronomy, The Johns Hopkins University, Baltimore, MD 21218 \\ budavari@pha.jhu.edu \\ István Csabai ${ }^{2}$ \\ Department of Physics, Eötvös University, Budapest, Pf. 32, Hungary, H-1518 \\ Andrew J. Connolly \\ Department of Physics and Astronomy, University of Pittsburgh, Pittsburgh, PA 15260 \\ and \\ Zlatan Tsvetanov \\ Department of Physics and Astronomy, The Johns Hopkins University, Baltimore, MD 21218
}

\begin{abstract}
We propose a novel type filter for multicolor imaging to improve on the photometric redshift estimation of galaxies. An extra filter - specific to a certain photometric system - may be utilized with high efficiency. We present a case study of the Hubble Space Telescope's Advanced Camera for Surveys and show that one extra exposure could cut down the mean square error on photometric redshifts by $34 \%$ over the $z<1.3$ redshift range.
\end{abstract}

Subject headings: galaxies: distances and redshifts — galaxies: photometry — instrumentation: miscellaneous

\section{Introduction}

The prediction of photometric redshifts (Koo 1985; Connolly et al. 1995a; Gwyn \& Hartwick 1996; Sawicki, Lin \& Yee 1997; Hogg et al. 1998; Wang, Bahcall \& Turner 1998; Yee 1998; FernándezSoto, Lanzetta, \& Yahil 1999; Weymann et al. 1999; Benítez 2000; Csabai et al. 2000; Budavári

\footnotetext{
${ }^{1}$ Department of Physics, Eötvös University, Budapest, Pf. 32, Hungary, H-1518

${ }^{2}$ Department of Physics and Astronomy, The Johns Hopkins University, Baltimore, MD 21218
} 
et al. 2000) is built upon global continuum features of the underlying galaxy spectrum such as the Balmer break at $4000 \AA$. The more precisely one can localize these features, the more accurate the redshift estimates become. However, traditional broadband filters were not designed to provide the best possible photometric redshifts, for several reasons. In theory, a set of filters optimal for photometric redshift estimation might be designed, but this would probably be substantially different from any current standard set. Here we do not intend to solve the generic problem of finding an optimal filter set for photometric redshift estimation; this study shows how conventional filter sets can be extended to yield better photometric redshifts.

In Section 1.1, we present the idea of an additional filter and look into why and how an extra measurement - sampling the same wavelengths - can improve significantly on photometric redshifts. In Section 2, the algorithm is developed and we make a case study of the Advanced Camera for Surveys of the Hubble Space Telescope. Based on our analysis, we propose a novel type of "broad-band" filter that can be effectively used for photometric redshifts.

\subsection{The idea}

The more general inversion problem of photometry in terms of redshift, type, and intrinsic luminosity (Koo 1985; Connolly et al. 1995a,b, 1999; Budavári et al. 1999, 2000; Csabai et al. 2000) involves the study of the Jacobian matrix of fluxes as a function of the physical parameters of galaxies. Our numerical simulations show that filter sets yield more precise estimates when continuum spectral features are located around the overlap of two bands-both fluxes change rapidly with redshift. One way to improve the redshift estimates is to increase the number of filters and use narrower bands. This would require too many exposures and yield a non-standard photometric system (Hickson et al. 1998). The other possibility is to put an intermediate width (IM) filter in a broad band, which would help the redshift estimation by improving the resolution. A single IM band can only help in a narrow redshift range, so additional, narrower filters are needed for each broad band. This approach could triple the resolution but will heavily increase the total exposure time at the same signal-to-noise ratio.

We propose combining multiple IM filters located in standard photometric bands into one "broadband" filter. We call this a multihump filter. The resulting new filter will be able to improve the redshift prediction (wavelength resolution) over a much wider redshift range and will only require a single additional exposure of comparable length.

How does this really work? Our approach makes a large difference for blue galaxies. The $4000 \AA$ break in their spectra shows up only as a small "bump" which makes the redshift estimation difficult. Let us consider the worst-case scenario, a toy model spectrum of a late-type galaxy that declines with wavelength as a power law and has as its only spectral feature the approximately $500 \AA$ wide bump. Without this feature, the colors would be the same at any redshift. The flux changes with redshift within a band depending on the shape of the filter. If its gradient is too small (e.g. flat 
as a top-hat), tiny photometric errors can yield large errors in the redshift prediction. In other words, while the bump is in a broad band, it is hard to tell precisely where it is. The measured flux in our new filter will change in redshift regions where the spectral feature is passing through one of the IM humps. These are the regions where the proposed filter contributes the most to the redshift estimation and where the original filter set has poor performance (small gradient). Red elliptical (E/S0) galaxies do not present a significant problem, because the strong discontinuity at $4000 \AA$ changes noticeably the fluxes as it passes through the bands with redshift. In this case the additional filter plays a subsidiary role, and the improvement of the accuracy is incremental.

\section{Filter design}

The actual throughput of a photometric system strongly depends on the instrument, different projects use different CCDs to gain more sensitivity in the desired wavelength range. The quantum efficiency of these devices set strict constraints on the observable wavelength regime. The filter set is selected to cover the available spectral interval according to science goals. The multihump filter is specific to a filter set, and its transmissivity is affected by the CCD's quantum efficiency (QE).

We have analyzed the photometric system of the Hubble Space Telescope's Advanced Camera for Surveys (ACS Ford et al. 1996) ${ }^{3}$. The two back-illuminated CCDs of the wide field channel are VIS-AR coated in order to optimize the response in the $I$ band, and thus the $U$-band sensitivity has been sacrificed. Mission specifications dictate the constraints on the multihump filter that is to extend the original set of the $g^{\prime}, r^{\prime}, i^{\prime}$ and $z^{\prime}$ bands, which span the entire observable wavelength range. No bluer or redder filter can be used, because the detector is not sensitive at shorter or longer wavelengths.

The framework of the algorithm is simple. We parameterize a fictitious filter curve and convolve it with the CCD's actual QE to obtain a realistic response function. Simulated photometric catalogs of fluxes and errors are generated using the extended filter set. The goodness of a particular filter set is assessed by computing the errors on the redshift estimates.

In the context of current filter manufacturing techniques (Offer \& Bland-Hawthorn 1998), a feasible parameterization of the additional filter's response function $(R(\lambda)$, see Figure 1) consists of at most two notch filters, plus a low and a high cutoff, as described by

$$
\begin{aligned}
R(\lambda)= & \frac{1}{\pi} \arctan \left(\epsilon \cdot\left(\lambda-\lambda_{\text {start }}\right)\right)-\frac{1}{\pi} \arctan \left(\epsilon \cdot\left(\lambda-\lambda_{\text {end }}\right)\right) \\
& -\exp \left[-\left(\frac{\lambda-\lambda_{1}}{\Delta \lambda_{1}}\right)^{p}\right]-\exp \left[-\left(\frac{\lambda-\lambda_{2}}{\Delta \lambda_{2}}\right)^{p}\right] .
\end{aligned}
$$

Parameters $\lambda_{\text {start }}$ and $\lambda_{\text {end }}$ are the limits of the filter, and $\epsilon$ determines the gradients, the rise of the edges. The values of $\lambda_{1}$ and $\lambda_{2}$ correspond to the locations of the notches and $p$ (an even number)

\footnotetext{
${ }^{3}$ http://adcam.pha.jhu.edu
} 
changes their shape.

The mock catalogs were generated based on spectral energy distributions (SEDs) derived from a physical interpolation scheme applied to the spectra of Coleman, Wu \& Weedman (1980), as described in Connolly et al. (1999) and Budavári et al. (2000). On a high resolution redshift grid

$\left(\delta z=5 \times 10^{-3}\right)$, a hundred objects were generated at each grid point with random SEDs. The continuous type parameter was scaled to yield a population of galaxies in which the probability of having a spectral type between Ell and Sbc was 10\%, Sbc-Scd 35\%, Scd-Irr 35\%, and bluer $20 \%$. The fluxes were modulated with $3 \%$ photometric errors taken from a Gaussian distribution. The quality of the photometric redshifts is determined by computing the rms of the estimates. The error is calculated for the desired redshift interval (or regions) meeting the actual project requirements - in this study, redshifts between $z=0.2$ and $z=1.2$.

The actual optimization problem has quite a few parameters (see Equation 1) to solve for, which makes it hard to numerically find the global minimum. It is also quite time-consuming to evaluate the underlying function; a new loop of the simulation has to be completed each time. Also extra constraints were added to the problem. To be able to use the very same filter from the ground for testing purposes or in follow-up observations, a design compromise due to the atmosphere has been made. In the actual optimization algorithm, one of the notches was always forced to include the $5577 \AA$ sky line. To map the entire parameter space, a linear search on a coarse grid was used to find the best possible filter candidates, and then from the those minima, a gradient method was utilized with discrete, higher resolution step-sizes.

\section{Discussion}

The resulting shape of the multihump is shown in Figure 2. This best configuration has two humps and its transmissivity peaks at the shortest possible wavelengths in the $g^{\prime}$ band and, at longer wavelength, in the $i$ ' band. Since the ACS filters look almost like triangles (as a result of the strong wavelength dependency in the CCD's QE), our additional filter tries to intensify the changes in colors, at the lowest, $z \sim 0.2$, and highest, $z \sim 1.2$, redshifts. On one side, the optimal multihump filter is essentially trying to compensate the lack of a ultraviolet filter by boosting the contrast at the edge of the $g$ ' band. In fact, the algorithm also finds an optimal solution of having a $U$ band, which is not a real option because of the CCD's QE (see Section 2). The location of the hump in the $i$ ' is clearly set by the upper redshift limit $z \lesssim 1.2$, when the Balmer break is at $\lesssim 8800 \AA$.

Figure 3 compares the performance of the original and extended filter sets by plotting the rms error as a function of redshift. The accuracy of photometric redshifts for the entire catalog is shown first. The prediction improves at all redshifts; the overall improvement is

$$
\mathcal{Q}=\frac{\sigma_{\text {orig }}^{2}-\sigma_{\text {ext }}^{2}}{\sigma_{\text {orig }}^{2}}=34 \% .
$$


Early- and late-type subsamples are shown in the middle and right panels of Figure 3, respectively. The E/S0 estimates clearly get better at the smallest redshifts, $z \gtrsim 0$, and at around $z \lesssim 1.2$, when the $4000 \AA$ break is in one of the humps. However, the redshift prediction also improves in another redshift region, around $z \sim 0.55$, as a result of the $\mathrm{H} \beta$ and $\mathrm{Mg}$ I absorption as it passes through the second hump. In according with our expectations, redshift estimates of the late-type galaxies change much more drastically then those estimated for early types.

The large number of parameters makes it difficult to estimate the errors or covariances. In Table 1, the rms errors computed for different set of filters are shown. The original ACS filter set gives $\sigma_{z}=0.075$. If we try additional multihump filters with humps centered on the $g^{\prime} r^{\prime} i$ ' and $g^{\prime} r$ ' $z$ ' bands, the performance just slightly improves; $\sigma_{z}=0.072$ and 0.073 , respectively. Centering humps on the gaps between the original bands (off-band multihump filter) does not improve on the accuracy of the estimates either; $\sigma_{z}=0.073$. The optimized filter yields much better redshifts, $\sigma_{z}=0.058$. The gain in the entire catalog is comparable to what would be achievable by using separate IM filters. The redshift accuracy can be marginally increased by splitting the optimal multihump into two separate filters $\left(\sigma_{z}=0.055\right)$, but the additional exposure time would be more than twice as long as in our approach.

\section{Conclusion}

We propose the use of multihump filters extending standard photometric systems to make photometric redshift estimation much more efficient. Our simulations have shown that an optimized extra filter could improve the redshift prediction by $34 \%$ for the Advanced Camera for Surveys. The improvement takes place over the $z<1.3$ redshift range. The filter's effective width is about $1500 \AA$, comparable to the existing photometric bands, so the measurement would require only about $25 \%$ more exposure time. Such a filter can be built with today's technology; in fact, similar filters have been utilized to suppres atmospheric features at near-infrared wavelengths (Offer \& Bland-Hawthorn 1998). The shape of the extra filter can be tuned to different goals and missions. It can be optimized for different filter sets and adjusted to desired redshift ranges.

I. C. and T. B. acknowledge partial support from the Hungarian Academy of Sciences-NSF grant 124 and the Hungarian National Scientific Research Foundation grant T030836, A. J. C. acknowledges support from NASA through a Long Term Space Astrophysics grant (NAG 5-7934) and through grant GO-07817.06-96A from the Space Telescope Science Institute. A. S. acknowledges

support from the NSF (AST 98-02980) and the NASA Long Term Space Astrophysics program (NAG 5-3503). 


\section{REFERENCES}

Benítez, N., 2000, ApJ, 536, 571

Budavári, T., Szalay, A.S., Connolly, A.J., Csabai, I. \& Dickinson, M.E., 1999, in Photometric Redshifts and High Redshift Galaxies, eds. R.J. Weymann, L.J. Storrie-Lombardi, M. Sawicki, \& R. Brunner, (San Francisco: ASP), 19

Budavári, T., Szalay, A.S., Connolly, A.J., Csabai, I. \& Dickinson, M.E., 2000, AJ, 120, 1588

Coleman, G.D., Wu., C.-C., \& Weedman, D.W., 1980, ApJS, 43, 393

Connolly, A.J., Csabai, I., Szalay, A.S., Koo, D.C., Kron, R.G., \& Munn, J.A., 1995a, AJ, 110, 2655

Connolly, A.J., Szalay, A.S., Bershady, M.A., Kinney, A.L., \& Calzetti, D., 1995b, AJ, 110, 1071

Connolly, A.J., Budavári, T., Szalay, A.S., Csabai, I., \& Brunner, R.J., 1999, in Photometric Redshifts and High Redshift Galaxies, eds. R.J. Weymann, L.J. Storrie-Lombardi, M. Sawicki, \& R. Brunner, (San Francisco: ASP), 13

Csabai, I., Connolly, A.J., Szalay, A.S., \& Budavári, T., 2000, AJ, 119, 69

Fernández-Soto, A., Lanzetta, K.M., \& Yahil, A., 1999, ApJ, 513, 34

Ford, H.C., et al., 1996, SPIE, 2807, 184

Gwyn, S.D.J., \& Hartwick, F.D.A., 1996, ApJ, 468, L77

Hickson, P., et al., 1998, SPIE, 3352, 226

Hogg, D.W, et al., 1998, AJ, 115, 1418

Koo, D.C., 1985, AJ, 90, 148

Offer, A.R., \& Bland-Hawthorn, J., 1998, MNRAS, 229, 176

Sawicki, M.J., Lin, H., \& Yee, H.K.C, 1997, AJ, 113, 1

Wang, Y., Bahcall, N., \& Turner, E.L., 1998, AJ, 116, 2081

Weymann, R.J., Storrie-Lombardi, L.J., Sawicki, M., \& Brunner, R., (editors), 1999, Photometric Redshifts and High-Redshift Galaxies (San Francisco: ASP)

Yee, H.K.C., 1998, in Proc. 10th Recontres de Blois, in press (astro-ph/9809347) 
Table 1. ERrors on Photometric Redshifts

\begin{tabular}{lccccccccc}
\hline \hline Filter Set & $\Delta z_{\text {rms }}$ & $\lambda_{\text {start }}$ & $\lambda_{\text {end }}$ & $\lambda_{1}$ & $\Delta \lambda_{1}$ & $\lambda_{2}$ & $\Delta \lambda_{2}$ & $p$ & $\epsilon$ \\
\hline Original & 0.075 & $\ldots$ & $\ldots$ & $\ldots$ & $\ldots$ & $\ldots$ & $\ldots$ & $\cdots$ & $\cdots$ \\
$g^{\prime} r^{\prime} i$ & 0.072 & 4800 & 7900 & 5700 & 450 & 7000 & 600 & 8 & 0.05 \\
$g^{\prime} r^{\prime} z$ & 0.073 & 4900 & 8900 & 5700 & 450 & 7550 & 900 & 8 & 0.05 \\
Off-band & 0.073 & 5200 & 8800 & 6200 & 400 & 7800 & 400 & 8 & 0.05 \\
Optimized & 0.058 & 4000 & 8500 & 6250 & 1750 & $\cdots$ & $\cdots$ & 20 & 0.05 \\
Separate & 0.055 & $\ldots$ & $\cdots$ & $\cdots$ & $\cdots$ & $\cdots$ & $\cdots$ & $\cdots$ & $\cdots$ \\
\hline
\end{tabular}

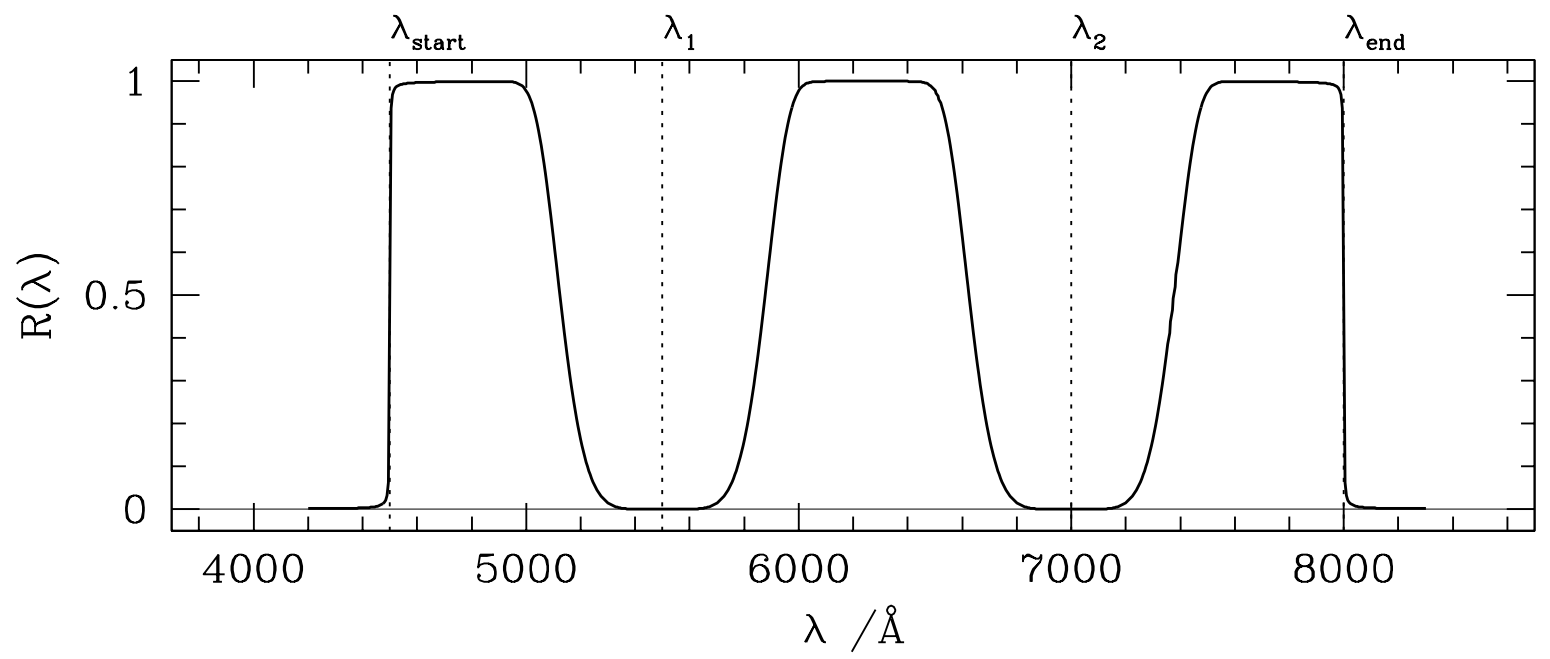

Fig. 1. - Illustration of the filter's response curve, as defined by Equation 1. 


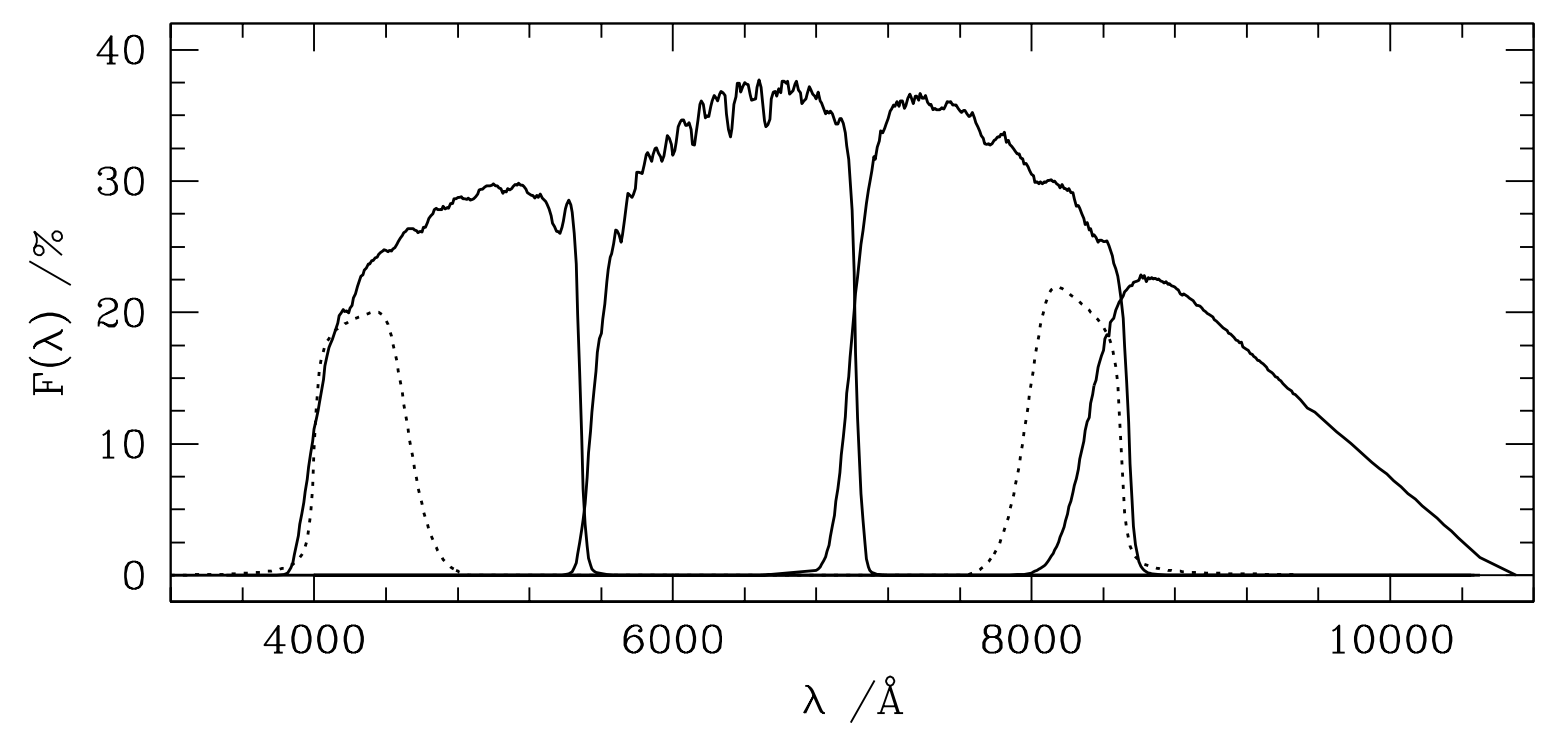

Fig. 2. - The throughput of the filters as used in convolutions. The original $g^{\prime}, r^{\prime}, i$, and $z^{\prime}$ bands are represented by solid lines (left to right). The add-on multihump filter (dotted line) is scaled down by $30 \%$ for illustration. 


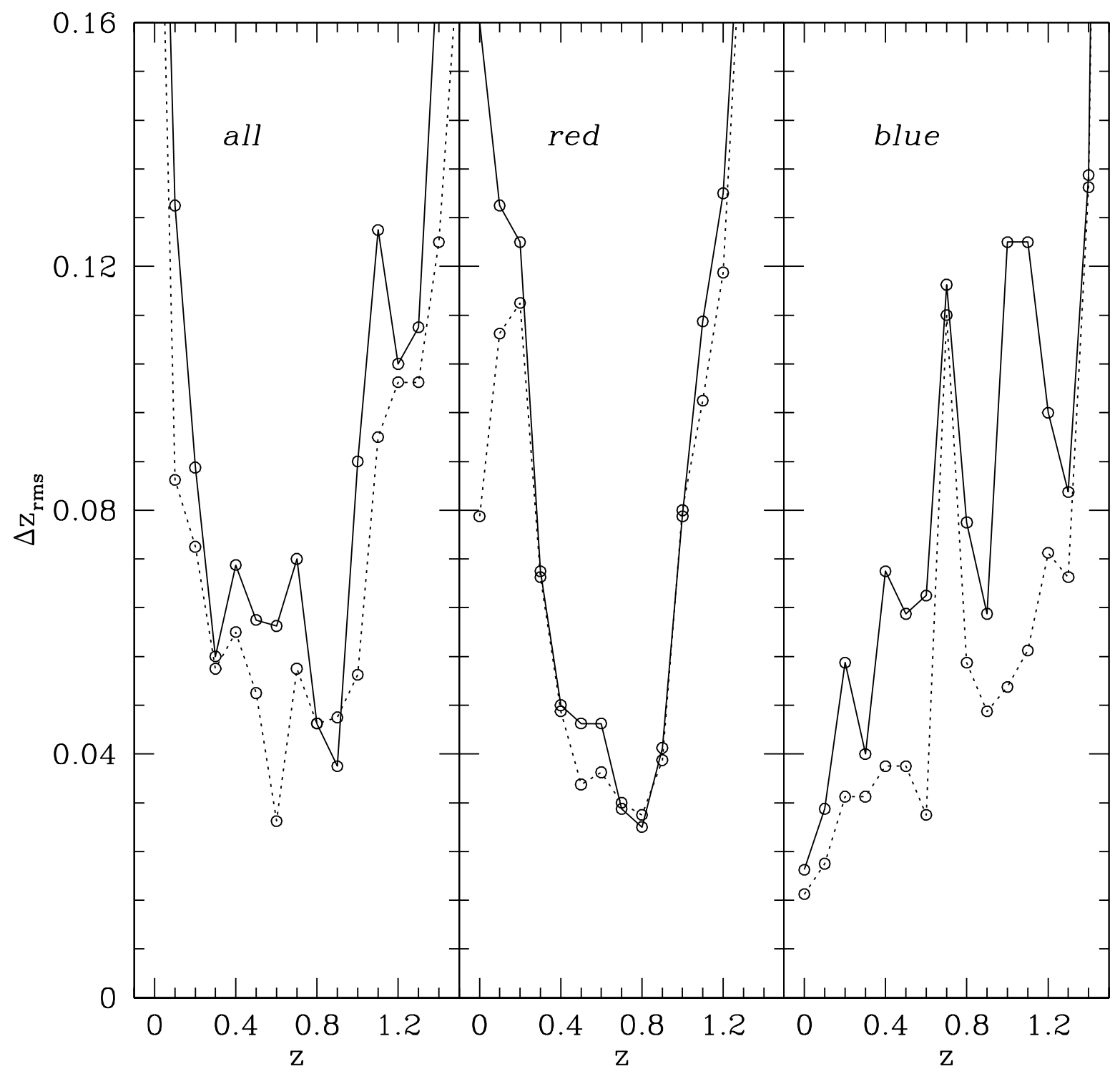

Fig. 3. - The rms error on photometric redshifts using the original filter set (solid line) and the extended set (dotted line). Left to right: The improvement for the entire population in the simulation and for the red (E/S0) and blue subsamples, respectively. The large errors at low redshifts are due to the lack of the $U$ band; at redshift $z>1$, one would need a $J$ band or higher. 